\title{
Análisis espacial de las dinámicas de crecimiento económico en México (1999-2009)
}

\section{Spatial analysis of economic growth dynamics in Mexico (1999-2009)}

\author{
Liz Ileana Rodríguez-GÁmez * \\ José Antonio Cabrera-Pereyra**
}

\begin{abstract}
This article is a first look into the exploratory spatial analysis of economic growth across Mexican municipalities over the period from 1999-2009. Through the exploratory spatial data analysis (ESDA) and geo-statistical techniques, the paper uncovers the importance of spatial contiguity in scattering the economic growth. Therefore, using a spatial and temporal perspective, the article analyzes the spatial autocorrelation phenomenon in identifying patterns and territorial dynamics of economic growth. Finally, it shows the municipalities with virtuous and vicious processes of growth or stagnation, as well as those with resilience to economic growth.
\end{abstract}

Keywords: economic geography, economic growth, spatial analysis, spatial autocorelation, spatial heterogeneity.

\section{Resumen}

Este artículo es un primer acercamiento al análisis exploratorio territorial del crecimiento económico municipal en México durante el periodo 1999-2009. A través del Análisis Exploratorio de Datos Espaciales (ESDA, por sus siglas en inglés) y el uso de técnicas geo-estadísticas se investiga la importancia de la proximidad espacial en la propagación del crecimiento. Por ello, con una perspectiva espaciotemporal se analiza el fenómeno de la autocorrelación espacial en la identificación de patrones y dinámicas territoriales de crecimiento económico. Finalmente, se localizan los municipios con procesos virtuosos y viciosos de crecimiento o estancamiento, así como aquellos con una resiliencia al crecimiento.

Palabras clave: geografía económica, crecimiento económico, análisis espacial, autocorrelación espacial, heterogeneidad espacial.

*El Colegio de Sonora, correo-e: 1rodriguez@colson.edu.mx

**University of Arizona, correo-e: jacp1104@gmail.com 


\section{Introducción}

En un principio, el análisis del vínculo entre geografía y economía se estudió haciendo alusión a elementos geográficos como el clima, la vegetación y una serie de condicionantes geográficas que tienen influencia en el crecimiento; sin embargo, en años recientes el estudio de esta relación ha adquirido una connotación metodológica diferente. Es decir, la reconsideración y revalorización del espacio en el análisis económico ha traído la aparición de un nuevo campo teórico que retoma los estudios pioneros de la teoría de la localización, la geografía económica y la ciencia regional. La síntesis de estos enfoques ha madurado en una nueva formulación metodológica que considera un conjunto de técnicas basadas en la geoestadística, la econometría espacial, la simulación matemática y los Sistemas de Información Geográfica (SIG).

Debido a lo anterior, el crecimiento económico regional es reconsiderado como un proceso espacial que se esparce por el territorio y está influenciado por el desempeño económico de las áreas vecinas, es decir, existe correlación espacial. Por ello, para conocer un poco más sobre cómo este proceso se contagia a regiones vecinas se realiza un Análisis Exploratorio de Datos Espaciales (ESDA, por sus siglas en inglés) para investigar la relación existente entre geografía y crecimiento económico. Así, el objetivo es realizar un diagnóstico espacial y temporal de la dependencia y la heterogeneidad en el crecimiento, los cuales lleven a la definición de dinámicas territoriales de crecimiento entre los municipios de México.

$\mathrm{Al}$ considerar a la proximidad geográfica (contigüidad) como uno de los elementos que potencia el proceso de crecimiento económico en los municipios de México, se realiza una exploración espacial del crecimiento en el periodo de 1999 a 2009. Este análisis permite identificar patrones y definir dinámicas de crecimiento en el territorio y en el tiempo. El análisis simultáneo en tiempo y espacio permite encontrar diferencias regionales en las dinámicas territoriales del crecimiento $\mathrm{y}$, en consecuencia, esbozar algunas recomendaciones de política regional.

Para la identificación de las dinámicas territoriales del crecimiento entre municipios mexicanos el artículo se integra en tres secciones, además de la presente introducción y los comentarios finales. En la primera sección se argumentan los aciertos e inconvenientes en el estudio del crecimiento económico, desde la perspectiva de la ciencia regional y a la luz de los nuevos planteamientos de la geografía económica. El segundo apartado introduce al tema de estudio a través de un diagnóstico de la dependencia espacial y de los patrones del crecimiento. De ahí que en la tercera sección se analice la heterogeneidad espacial y se realice una exploración geográfica para identificar las dinámicas de crecimiento. Finalmente, se resumen 
los hallazgos de las dinámicas territoriales del crecimiento en los municipios de México en el periodo 1999-2009.

\section{La economía espacial y el crecimiento económico}

En los años posteriores a la segunda guerra mundial y tras la nueva lectura de la teoría clásica alemana de la localización, surgida en el siglo XIX, emergió la economía espacial; ésta última se institucionalizó más tarde como ciencia regional (Isard, 2003; Boyce, 2004). El desafío que implicó dar a conocer los fundamentos de esta ciencia fue mayor cuando las herramientas analíticas disponibles resultaron insuficientes para comprender la complejidad de la relación entre economía y espacio, y apoyar así su avance. De ahí que la economía espacial tenga sus antecedentes como conocimiento sistemático en los incipientes trabajos de localización espacial que se desarrollaron en la época (Ponsard, 1983), y con el soporte de la ciencia regional (Isard, 2003) y la geografía económica (Barnes, 2001).

La ciencia regional inició con los trabajos de economistas, llamados científicos regionales; a medida que ésta se difundía adquirió mayor aceptación entre "geógrafos economistas", quienes desde los ochenta han aumentado sus publicaciones en esta área (Florax y Plane, 2004; Rey y Anselin, 2000). Sin embargo, tras la revolución cuantitativa en la ciencia regional y en la geografía económica en particular (Barnes, 2002) -además del desarrollo de las computadoras y paquetes informáticos en matemáticas, estadística y geografía-la disciplina de la economía espacial cobró un nuevo auge. Con nuevos métodos y técnicas de medición de la relación y el efecto del espacio en la economía, el análisis espacial empezó a aplicarse en la solución de problemas regionales y urbanos.

Específicamente, la economía espacial se ocupa del análisis de las cuestiones: qué hay, dónde y por qué; es decir, estudia los tipos específicos de actividades económicas, su localización en relación con otras actividades, se cuestiona los problemas relativos a la proximidad, la concentración y la dispersión de las actividades, así como las semejanzas y diferencias de los patrones de distribución geográfica (Hoover y Giarratani, 1984). Además, tras el redescubrimiento de la geografía por los economistas, la Nueva Geografía Económica (NGE) incluyó en su enfoque metodológico técnicas de análisis espacial que permiten modelar, establecer conexiones y discutir temas relacionados con la aglomeración en el espacio geográfico.

Dentro de estas nuevas técnicas de análisis, el desarrollo de la geoestadística, la econometría espacial y los SIG son la base para el análisis exploratorio de datos espaciales. El ESDA es una colección de técnicas que permite describir y visualizar la distribución espacial de un fenómeno descubriendo patrones de asociación espacial, identificando aglomeracio- 
nes -clústeres o hotspots- y sugiriendo regímenes espaciales (Anselin et al., 2007). Esta exploración de datos y su distribución en el espacio geográfico permite, a través de gráficas, mapas y medidas de asociación espacial, conocer el fenómeno de estudio en la explicación del por qué ocurre la aglomeración en una localización específica, qué implicaciones hay para las áreas vecinas y viceversa.

En años recientes, el fenómeno de la aglomeración se ha relacionado estrechamente con la distribución espacial de la inequidad, la cual ha sido tema central en varias disciplinas de las ciencias sociales. La existencia de núcleos de concentración de actividades generadoras de riqueza rodeadas por anillos de pobreza se relaciona directamente con el estudio territorial de los fenómenos del desarrollo económico (Goodchild et al., 2000).

Dicha concentración de la riqueza se desdibuja a distintos niveles de agregación geográfica (Fujita y Tomoya, 2005), por lo que una concentración inicial del ingreso en cierta región es atribuible a una desigual dotación de recursos naturales, infraestructura social y económica, localización de la producción y del consumo, así como flujos de comercio; con el tiempo este hecho ejerce una atracción de fuerza de trabajo de regiones periféricas hacia el centro, lo cual genera, en un primer momento,un rápido crecimiento económico y posteriormente una desaceleración que eventualmente disminuye las diferencias de ingreso entre regiones.

Es por ello que geográficamente se observan patrones espaciales en la distribución de la actividad económica y en procesos de aglomeración territorial; de ahí que los estudios económicos se han enfocado al análisis de las condiciones geográficas del crecimiento y el desarrollo, siempre en función de factores ligados a la localización de la producción (Krugman, 1998). Resulta claro que, como resultado de las dinámicas espaciales, existe una interconectividad entre distintos espacios, sus estructuras productivas y el crecimiento económico; los efectos de una región sobre otra se propagan espacialmente, en ese sentido, no se busca entender el efecto de la aglomeración en el crecimiento y desarrollo económico de una localización dada, sino cómo ésta afecta a otras aglomeraciones y viceversa; por tanto el énfasis en el espacio económico se desplaza de la especialización a la complementariedad entre distintos espacios geográficos.

El concepto clave detrás del análisis de las dinámicas del crecimiento económico es el de autocorrelación espacial, cuyo planteamiento teórico retoma la primera ley de la geografía, la cual argumenta que todos los elementos están relacionados entre sí, pero los cercanos en el espacio tienden a ser más similares o estar más relacionados que aquéllos más distantes (Tobler, 1970). En consecuencia, la autocorrelación espacial mide el grado de dependencia o correlación de una variable que es atri- 
buida a la proximidad de dichos valores en el espacio geográfico (Cliff y Ord, 1970; Griffith, 2003).

$\mathrm{Al}$ respecto, avances en pruebas estadísticas para medir la autocorrelación espacial se habían desarrollado décadas atrás por Moran (1948) y Geary (1954), sin embargo, el cálculo de dichos estadísticos se dificultaba al considerar un conjunto mayor de observaciones o áreas geográficas. No es sino hasta la década de los noventa, con el desarrollo de software especializado (Anselin, 2000), que el estudio de la autocorrelación espacial se popularizó como técnica de análisis de los fenómenos que tienen alguna incidencia en el espacio.

Si bien, los estudios de las desigualdades económicas entre regiones y sus implicaciones espaciales se desarrollaron tardíamente con la propuesta de Barro y Sala-i-Martin (2004), en relación con las dinámicas de crecimiento económico y la convergencia regional (Rey, 2004), la riqueza de la dimensión geográfica inmersa en el tema y el estudio de la interacción espacial no habían sido punto focal en el análisis del crecimiento y la convergencia hasta antes de la aplicación de las técnicas de análisis espacial.

En este sentido, la crítica espacial a la teoría económica neoclásica ha sido la omisión de los diferenciales geográficos en la dotación de factores de un lugar en particular y su efecto sobre las variables económicas en general y del crecimiento económico en particular, así como del efecto de todas aquellas áreas geográficas colindantes y no tan colindantes con las que pueda existir un nexo.

Es decir, para una mejor comprensión de dichos procesos es necesario considerar la proximidad y los vínculos entre regiones vecinas, lo anterior implica considerar la dependencia espacial en el crecimiento económico, es éste uno de los dos fenómenos estudiados por la economía espacial (Anselin, 1988; Abreu et al., 2005; Ertur y Le Gallo, 2009).

Además, existen implicaciones teóricas interesantes, pues la introducción del espacio como factor explicativo del crecimiento permite imponer una horizontalidad a un análisis que previamente sólo se estudiaba de manera vertical o temporal (Fingleton y López-Bazo, 2006). Así, las diferencias en la estructura de las economías regionales introducen la posibilidad de que exista heterogeneidad espacial: el segundo de los fenómenos estudiados por la economía espacial. Este fenómeno significa que, en el caso del crecimiento económico, el proceso no es estable en el territorio (Anselin, 1988). 


\section{Datos y métodos para el análisis espacial}

\subsection{El análisis exploratorio de datos espaciales}

Para un análisis diagnóstico que permita conocer las dinámicas de crecimiento económico entre los municipios de México, el ESDA permitirá una primera aproximación al fenómeno de crecimiento económico y a la manera como éste se propaga en el espacio geográfico. El ESDA surge a fines de los ochenta y se consolida en los noventa como un conjunto de técnicas destinadas a detectar esquemas de asociación espacial, concentraciones locales y regímenes espaciales presentes en un conjunto de datos para los cuales sus características de localización resultan esenciales (Anselin, 2000).

Así, al analizar un fenómeno de propagación con un fuerte componente social $\mathrm{u}$ otras características que resulten en una distribución segregada geográficamente, como es el caso del crecimiento económico, conviene preguntarse la forma en que éste se irradia a través de las unidades espaciales, y si tal conducta corresponde a algún modelo de difusión conocido o bien registra la segregación espacial de alguna característica. Para ello, el análisis de la autocorrelación espacial resulta conveniente, ya que refleja el grado en que objetos o actividades en una unidad geográfica son similares a los objetos o actividades en unidades geográficas próximas.

En la práctica, entre los indicadores para medir el grado de dependencia espacial frecuentemente referidos en la literatura de los SIG se encuentra la I de Moran (1948), la C de Geary (1954) y la G(d) de Getis y Ord (1992). Sin embargo, uno de los indicadores comúnmente utilizados para medir la $\mathrm{AE}$ o el grado de dependencia entre unidades espaciales es el Índice de Moran, ya que éste tiene una simple aplicación y adopta el principio estadístico del coeficiente de correlación de Pearson, pero incorporando una medida de proximidad entre los elementos. La I de Moran considera el nivel de asociación lineal entre los valores observados y sus valores rezagados en el espacio (Moran, 1948; Anselin, 2007), y también se le conoce como un indicador de autocorrelacion "global", ya que concentra en una sola cifra el patrón de distribución territorial del fenómeno bajo estudio. La expresión algebraica del índice está definida en la ecuación (1):

$$
I=\frac{N \sum_{i} \sum_{j} w_{i j}\left(x_{i}-\bar{x}\right)\left(x_{i}-\bar{x}\right)}{\left(\sum_{i} \sum_{j} w_{i j}\right) \sum_{i}\left(x_{i}-x\right)^{2}}
$$

Donde $N$ es el número total de áreas, $\mathrm{w}_{\mathrm{ij}}$ captura la proximidad espacial (contigüidad) entre las áreas $i$ y $j$ en una matriz de pesos, $x_{i}$ y $x_{j}$ son los 
valores observados de una variable (autocorrelación espacial univariada) o de una segunda variable (autocorrelación espacial bivariada) para las áreas $i$ y $j$ respectivamente, y $\bar{x}$ es el promedio de los valores. Como se señaló, es necesario considerar la relación que existente entre la variable analizada y la geografía, es decir, el peso o grado de influencia de los municipios entre sí (Abreu et al., 2005). La proximidad espacial, entonces, conecta a un conjunto de unidades contiguas mediante algún criterio espacial previamente establecido (Baumont et al., 2004) ${ }^{1}$. Ya que la matriz de pesos se selecciona exógenamente, se siguieron las recomendaciones hechas por Anselin $(2000 ; 2003)$ y Abreu et al. (2005) para seleccionar el criterio a utilizar en la definición del arreglo espacial ente los municipios.

Por consiguiente, si los valores del estadístico son mayores (menores) al valor esperado, se indica una autocorrelación espacial positiva (negativa) o similitud (diferencia) entre las regiones vecinas. De acuerdo con lo anterior, el estadístico adopta valores en el rango de $-1 \mathrm{a}+1$. La interpretación del indicador es similar a la de un coeficiente de correlación, por lo que un valor cercano a cero indica que no existe autocorrelación, es decir, una distribución espacial aleatoria o independencia en la distribución territorial de la variable; mientras que el signo sugieren una autocorrelación espacial negativa o positiva. El indicador cuenta además con una construcción estadística que permite decidir si el patrón espacial (autocorrelación negativa o positiva) es diferente de cero, es decir, si se desvía significativamente de una distribución aleatoria.

La asociación significativa entre localización y los valores de referencia puede no estar presente en todo el espacio analizado, sino sólo en determinadas zonas, por lo que son necesarios indicadores locales de $\mathrm{AE}$, que tienen como objetivo que el estadístico obtenido para cada zona suministre información acerca de la relevancia de valores similares alrededor de la misma. Los más utilizados son la G(d) de Getis y Ord (1992) y la I de Moran de Anselin (1995). De nuevo por simplicidad y seguimiento a nuestro análisis, el estadístico local de la I de Moran se define en la ecuación (2):

$$
I=\frac{x_{i}-\bar{x}}{s_{x}^{2}} \sum_{j}\left[w_{i j}\left(x_{j}-\bar{x}\right)\right]
$$

Donde $s_{x}^{2}=\sum_{j}^{n}\left(x_{i}-\bar{x}\right) / n$ es la varianza y el resto de la notación es similar a la descrita en la ecuación 1, es posible también un análisis de la autocorrelación espacial bivariada. Una manera de visualizar e identificar geo-

\footnotetext{
${ }^{1}$ El arreglo espacial puede definirse a través de: 1) las combinaciones que se establecen en un tablero de ajedrez (e.g. queen, rook), 2) la determinación a priori del número de regiones vecinas (i.e. $k$ nearest neighbor) y 3) el criterio de distancia física con un cut-off en donde al menos cada región tenga un vecino.
} 
gráficamente los agrupamientos es a través de un mapa de indicadores locales de asociación espacial (LISA, por sus siglas en inglés), el cual contiene cuatro posibles agrupaciones en la identificación de clústeres estadísticamente significativos: áreas BB (Bajo-Bajo) y áreas AA (AltoAlto) que contienen unidades geográficas con bajos o altos valores de la variable rodeados por bajos o altos valores; además de áreas BA (BajoAlto) y áreas AB (Alto-Bajo) que contienen observaciones de bajos valores rodeadas por áreas de altos valores y viceversa. Por ello, si el indicador de la I de Moran local es positivo, se sugiere el agrupamiento de unidades con atributos similares (AA y BB), en caso contrario los valores apuntan a un agrupamiento de unidades geográficas disímiles (AB y BA) (Anselin, 2003).

\subsection{Fuentes de información para el análisis}

Como se mencionó en secciones anteriores, el crecimiento económico es una variable que teóricamente muestra una dependencia espacial y podría presentar patrones de segregación en el espacio geográfico; para conocer al respecto, el análisis espacial permite una exploración inicial y una prueba estadística que muestra los patrones de comportamiento y dinámicas espaciales del crecimiento económico a escala global y local. Para ello, el primer paso consiste en construir una base de datos espaciales sobre el crecimiento económico a nivel de municipios, los cuales serán nuestra unidad espacial de análisis.

Así, con información de los Censos Económicos y de los Censos de Población del Instituto Nacional de Estadística y Geografía (Inegi, 2015a; 2015b; 2015c; 2015d; 2015e; 2015f) se construyó una variable proxy del desarrollo económico municipal, se consideró la trasformación logarítmica del valor agregado per capita ${ }^{2}$ y posteriormente se calculó la tasa anual de crecimiento económico ( $\triangle$ VAPCP) en la década de 1999 al 2009 y sus dos periodos quinquenales (1999-2004 y 2004-2009). ${ }^{3} \mathrm{Al}$ respecto, la econometría del crecimiento señala que en la medición de los niveles de desarrollo económico, el uso de una tasa de crecimiento per cápita o por trabajador suele ser indistinta, siempre y cuando la participacion de los trabajadores haya sido estable en el periodo analizado, como ocurre en el corto plazo (Durlauf et al., 2005).

${ }^{2}$ Adicionalmente, la información fue deflactada por la paridad del poder de compra a precios del año 2000, la cual no observó cambios sustantivos al trabajar con regiones dentro de un mismo país, ya que el indicador de precios no logra capturar las disparidades en el poder de compra a un nivel desagregado.

${ }^{3}$ Esta información se unió a la cartografía municipal del Marco Geoestadístico Nacional, que corresponde a la versión 5.0 A del Censo de Población y Vivienda 2010, en congruencia con el periodo de análisis de los datos. 
Si bien este indicador de desarrollo económico, y la subsecuente tasa de crecimiento calculada, omite información del nivel de actividad económica informal, subestimando los niveles de desarrollo economico, tiene la ventaja de proveer información representativa de las unidades económicas más dinámicas de la actividad productiva en cada municipio (Aguayo y Salas, 2002). No obstante, otros métodos resultan también efectivos para las estimaciones del crecimiento económico, a través de ellos es posible realizar estimaciones del Producto Interno Bruto (PIB) a nivel municipal, las cuales capturan las características esenciales del sistema económico y muestran la estructura productiva nacional.

\section{La geografía del crecimiento económico en México}

\subsection{La distribución territorial del crecimiento económico municipal}

México se ha caracterizado por su inequidad regional, la cual se enmarca en un gran número y diversidad de municipios. Al respecto, estudios sobre crecimiento económico, realizados con un enfoque espacial, señalan que efectivamente existe heterogeneidad regional en México, ante la presencia de una creciente polarización y el surgimiento de una desigualdad entre las regiones del país de naturaleza cada vez más compleja (Sastré-Gutiérrez y Rey, 2008; Rey y Sastré-Gutiérrez, 2010). Los estudios evidencian, a nivel de entidad federativa, la recurrente presencia de periodos de aumento en la dispersión del ingreso, los cuales se acompañan de incrementos en la heterogeneidad a nivel nacional.

No obstante, una rápida mirada a la distribución geográfica del crecimiento económico a nivel municipal da cuenta también de tal heterogeneidad. A nivel municipal, al parecer, hay una tendencia a la concentración del desarrollo económico, sin cambios importantes al finalizar el periodo de estudio; el mapa 1 permite visualizar qué niveles de desarrollo económico similares se registraron en municipios del norte de México, en la región centro-occidente y en las costas de la Península de Yucatán. En cambio, la distribución geográfica de la tasa de crecimiento por quinquenios tiene un patrón de propagación más ampliamente extendido y difuso para definir una pauta territorial de propagación geográfica del crecimiento económico (mapa 2). Comparativamente, hay cambios significativos en la distribución del crecimiento entre quinquenios; por ejemplo, en el norte de México varios municipios crecieron a menores tasas en el segundo quinquenio, mientras que los municipios del sur crecieron a mayores tasas. 


\section{Mapa 1 \\ Distribución por cuartil de los niveles de desarrollo económico municipal en México}

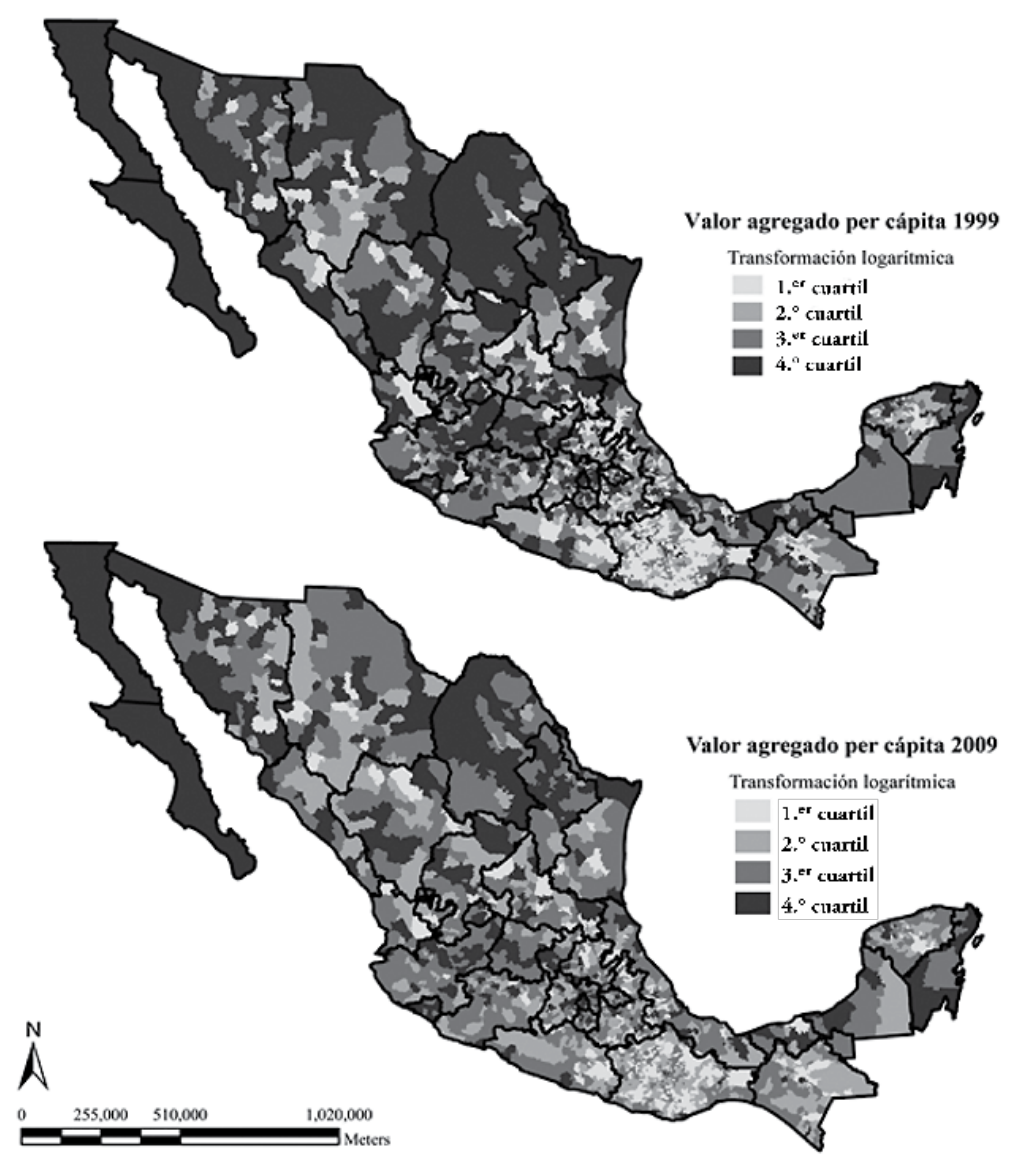

Fuente: elaborado con base en datos de los Censos Económicos de 1999 y 2009 (Inegi, 2015a; 2015c) y Censos de Población del 2000 y 2010 (Inegi, 2015d; 2015f). 


\section{Mapa 2}

\section{Distribución por cuartil de la tasa de crecimiento} económico municipal

Valor agregado per cápita (VAPCP)

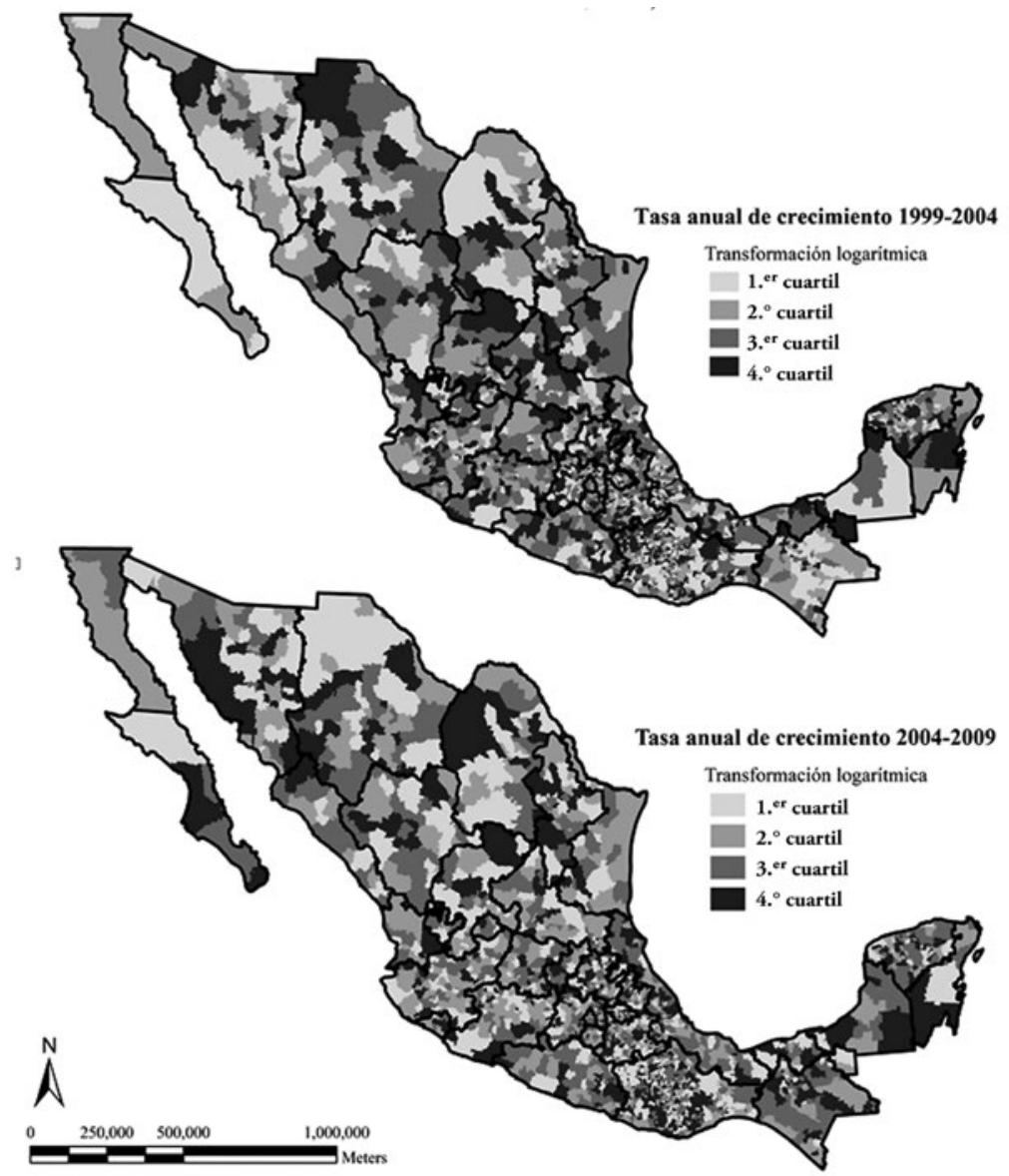

Fuente: elaborado con base en cálculos de la tasa de crecimiento del valor agregado per cápita con información de los Censos Económicos de 1999, 2004 y 2009 (Inegi, 2015a; 2015b; 2015c) y Censos y Conteos de Población del 2000, 2005 y 2010 (Inegi, 2015d; 2015e; 2015f). 
La exploración geográfica de la información sugiere que un fenómeno de concentración de los niveles de desarrollo económico tuvo lugar pero desconocemos dónde ocurrió esto y qué municipios o regiones estuvieron involucrados en este proceso; menos aun contamos con evidencia de cómo se registró el cambio en el patrón geográfico de propagación del crecimiento a través del tiempo.

Por ello, un análisis ESDA permite identificar y concluir si existió una tendencia hacia la aglomeración, en donde la densidad de municipios con alto desempeño económico varía significativamente de una región a otra, o si efectivamente el crecimiento económico fue aleatorio -sin una tendencia dominante hacia la aglomeración o la dispersión- o, en cambio, si este crecimiento fue disperso, donde municipios con alto crecimiento económico aparecerán uniformemente distribuidos en el espacio geográfico.

\subsection{Los patrones territoriales de crecimiento económico}

La evidencia presentada da cuenta que los municipios cercanos tienden a registrar características similares a las de sus vecinos, es decir, se observa una dependencia espacial. Por ello, con el fin de verificar estadísticamente la presencia de autocorrelación espacial en los niveles de desarrollo y crecimiento económico en los municipios de México, se calculó el estadístico global de la I de Moran.

El análisis de autocorrelación global permite concluir, con suficiente evidencia estadística, que el desarrollo económico tiene un patrón definido hacia la aglomeración, situación ya sugerida en el mapa 1. Se observa con el tiempo que la autocorrelación espacial disminuye pero continua siendo positiva y significativa; los valores del estadístico fueron 0.433, 0.419 y 0.304 para los ańos 1999,2004 y 2009 , respectivamente. ${ }^{4}$

En cambio, la distribución geográfica del crecimiento económico registró un patrón territorial aleatorio, ya que los valores del estadístico son pequeńos y cercanos a cero: 0.062 y 0.055 para el primero y segundo quinquenio, respectivamente, y 0.032 para la década de estudio. Pese a que puede registrarse una muy ligera tendencia a la concentración de municipios con patrones similares de crecimiento, al comparar entre quinquenios, el patrón general en la distribución territorial de la tasa de crecimiento económico continuó siendo aleatorio. Si bien el errático comportamiento del crecimiento económico sugiere una débil presencia del fenómeno de autocorrelación espacial ello no significa, de acuerdo

\footnotetext{
${ }^{4}$ Se empleó una matriz queen de contigüidad y el nivel de significancia estadística de las estimaciones fue de $99 \%$ con 4,999 permutaciones.
} 
con Florax y Nijkamp (2003), que se afecte la dependencia entre el crecimiento y la proximidad geográfica entre las áreas.

\section{Dinámicas territoriales de crecimiento económico}

\subsection{La dimensión espacial}

A consecuencia de una dotación inequitativa de factores productivos entre municipios, así como por diferencias en los niveles de productividad, algunos clústeres geográficos emergen en el territorio dando lugar a una heterogeneidad espacial en el crecimiento económico. Para analizarla se adopta una perspectiva local de la autocorrelación y se estiman indicadores locales de asociación espacial. Éstos permiten visualizar si los municipios con alto o bajo crecimiento están concentrados en algunas regiones, más que en otras, con lo cual se detectan zonas con tendencia a la agrupación o hotspots (Abreu et al., 2005). Esta herramienta es muy útil en la identificación de agrupamientos o clústeres, especialmente cuando no existe una idea a priori de la localización de éstos o de sus miembros.

Este análisis es compatible con el hecho de que el crecimiento, en su dimensión territorial, se disemina más allá o se contagia hacia áreas más extensas del territorio en función de las interdependencias existentes, dando como resultado el surgimiento de agrupamientos (i.e. clústeres). Así, por ejemplo, un municipio o un grupo de municipios pueden ser más o menos prósperos que las economías que los rodean, de tal manera que crecen más que el promedio de la región y su contribución a la economía regional es lo suficientemente significativa para distinguirse de los municipios contiguos que conforman su región. Con base en lo anterior se definen cuatro dinámicas territoriales para los municipios de México, identificándose su tendencia al crecimiento o estancamiento. ${ }^{5}$

En el mapa 3 se visualizan los resultados de los agrupamientos LISA observándose que las dinámicas territoriales pueden registrarse en dos situaciones: en un contexto de aislamiento territorial, ya que la autocorrelación espacial en municipios contiguos no resultó estadísticamente significativa; o bien, conformando agrupamientos de municipios vecinos donde se mezclan varias dinámicas. Por ello conviene analizar ambas situaciones por separado. En primer lugar se consideran los casos de municipios aislados, para los cuales se identificó su dinámica con base en las agrupaciones LISA.

${ }^{5} \mathrm{La}$ tendencia al estancamiento de un municipio se define a partir de una tasa de crecimiento menor al promedio de la región, es decir, menor al conjunto de vecinos contiguos a éste. No siempre el estancamiento se asocia a un crecimiento económico negativo. Como información adicional, tan sólo $11.7 \%$ del total de municipios de México registró una tasa anual negativa del desarrollo económico en la década de estudio. 


\section{Mapa 3 \\ Heterogenidad espacial en el crecimiento económico municipal de México}

(Transformación logarítmica de la tasa anual de crecimiento económico)

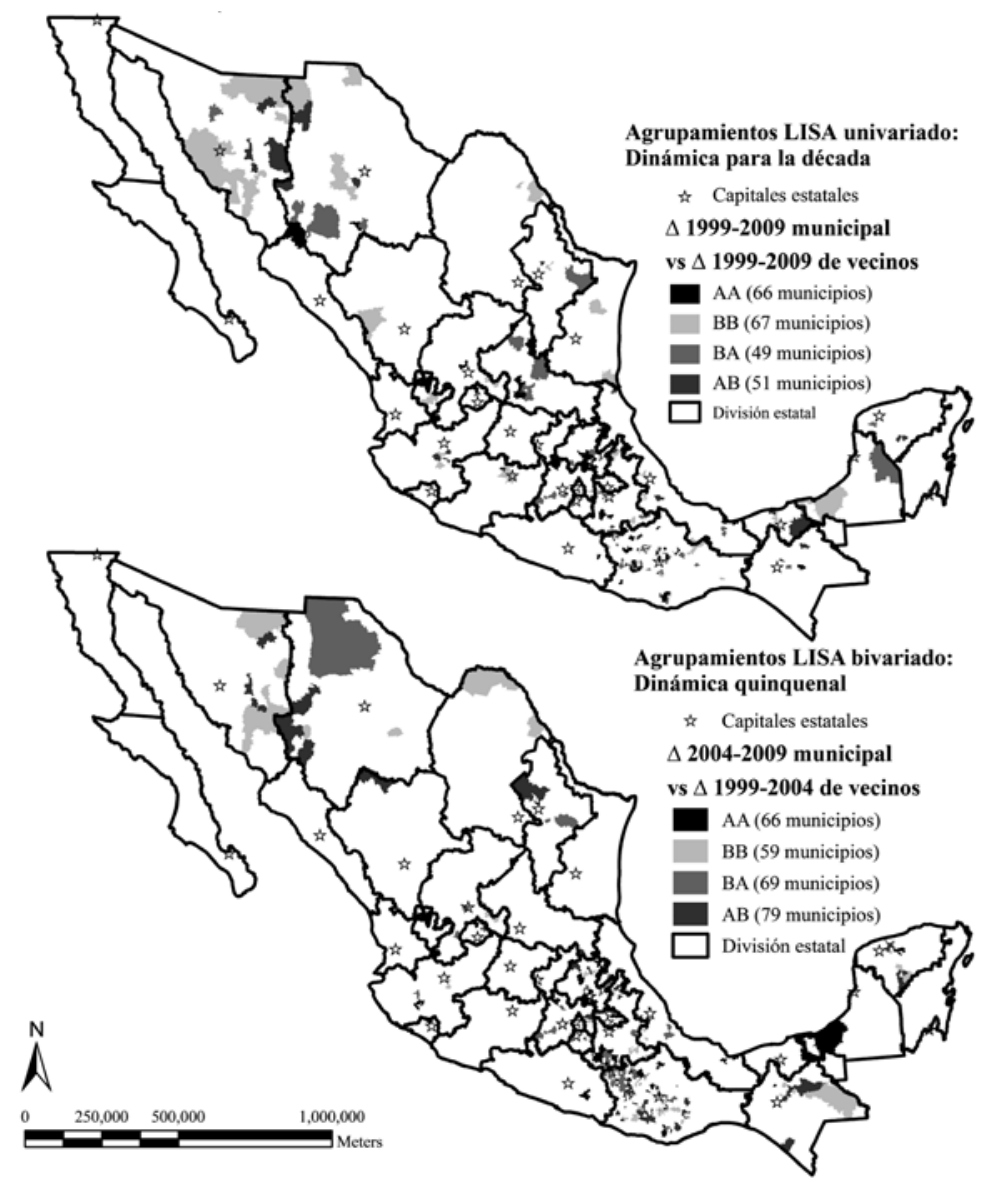

Nota: sólo reportan valores de la I de Moran Local (LISA por sus siglas en inglés) estadísticamente significativos al 5 por ciento.

Fuente: estimaciones de la heterogeneidad espacial local de la tasa de crecimiento económico en OpenGeoDa (v.1.2.0), con datos de los Censos Económicos de 1999, 2004 y 2009 (Inegi, 2015a; 2015b; 2015c) y Censos y Conteos de Población del 2000, 2005 y 2010 (Inegi, 2015d; 2015e; 2015f). 
a) Dinámica virtuosa de crecimiento económico (AA): es la dinámica de crecimiento óptima en donde el crecimiento de un municipio se contagió a sus municipios vecinos quienes, además de haber registrado un alto crecimiento económico, conformaron un clúster de crecimiento en la región. Se dieron también situaciones de municipios aislados sin efectos a sus áreas vecinas que funcionan como enclaves, casos como éstos se localizaron en:

1. Zacatecas: el municipio de Calera localizado en el corredor Zacatecas-Fresnillo.

2. Veracruz: municipios de la ZM de Coatzacoalcos, además de la ZM de Veracruz-Boca del Río y de la zona al sur de Poza Rica (Coatzintla y Coxquihui).

3. Guerrero: el municipio rural de Atlamajalcingo del Monte, en la región Montaña.

4. Oaxaca: algunos municipios que se encuentran en la región Cañada y en el distrito Sola de Vega en la región Sierra Sur de Oaxaca; en el distrito de Teposcolula en la región mixteca (San Pedro Nopala, Teotongo, San Antonio Acutla, La Trinidad Vista Hermosa y Santa Magdalena Jicotlán), el municipio de San Juan Colorado (distrito de Jamiltepec en la región Costa) y en el enclave que constituye la ciudad de Oaxaca.

5. Chiapas: Huixtán, contiguo a San Cristóbal de las Casas (región de Altos Tzotzil-Tzeltal).

b) Dinámica viciosa de crecimiento económico (AB): en los casos en que la economía del municipio no se integró al resto de la economía regional, el efecto territorial puede ser negativo, se registró un crecimiento municipal que no se propaga en la región. Si el crecimiento permanece encapsulado en sólo un municipio, mientras que el resto de las economías municipales registran bajos niveles de crecimiento en comparación al promedio de la región, la dinámica de crecimiento será "viciosa", es decir, basada en enclaves económicos que no beneficia a la economía regional que lo rodea. En esta situación se encuentran los siguientes municipios:

1. Nuevo León: el municipio de Ciénega de Flores, al norte de la ZM de Monterrey.

2. Veracruz: Chalma y Ayahualulco, al sur de Coatepec.

3. Oaxaca: San Miguel Tequixtepec (distrito de Coixtlahuaca en región mixteca), San Agustín Etla (distrito de Etla en región 
Valles Centrales) y San Miguel del Río (distrito de Ixtlán en región Sierra Norte).

4. Chiapas: Tumbalá (en la región de Tulijá Tzeltal Chol) y Soyaló (contiguo a la ZM de Tuxtla Gutiérrez, en la región de Bosques).

5. Yucatán: municipios de la región sur del estado como Tixméhuac y Tahdziú.

c) Dinámica viciosa de estancamiento económico (BB): municipios que se caracterizaron por presentar bajos niveles de crecimiento económico (i.e. por debajo de la media e incluso tasas negativas de crecimiento) y estar rodeadas por municipios vecinos en la misma situación. Si bien en estos municipios lo anterior podría no representar de inmediato un problema mayor, en el mediano y largo plazo esta dinámica de estancamiento podría llevar a una crisis o emergencia económica regional que, como efecto perverso territorial, contagie el estancamiento o una crisis económica a los vecinos de la región. En ella se encuentran municipios en:

1. Jalisco: en la región Norte del estado de Jalisco (Villa Guerrero y Bolaños).

2. Chihuahua: al norte del estado, en el municipio de Juárez, cabecera en Ciudad Juárez.

3. Durango: San Dimas en la región serrana (Tayoltita es la cabecera municipal).

4. Coahuila: los municipios de Guerrero y Allende en la región fronteriza del estado.

5. Nuevo León: el municipio de Marín, al occidente de la periferia de la $\mathrm{ZM}$ de Monterrey.

6. Oaxaca: Silacoyoapan y Calihuala (distrito de Silacoyoapan en la región mixteca), Santa María Coyotepec (distrito Centro en la región Valle Central), Santiago Yaveo (distrito de Choapan en la región del Papaloapan), Unión Hidalgo (distrito de Juchitán en la región Istmo).

7. Puebla: Zapotitlán en la región de Tehuacán y Sierra Negra.

8. Campeche: el municipio del Carmen; es Ciudad del Carmen la cabecera municipal.

d) Dinámica virtuosa de estancamiento económico (BA): situación en que municipios de bajo crecimiento estuvieron rodeados por otros con crecimiento económico por encima del promedio (municipios BA). Sin embargo, en este caso el estancamiento de un 
municipio se considera "virtuoso" ya que éste no se contagió a sus vecinos quienes, por el contrario, mantienen un crecimiento económico alto. Por ello, en el corto y mediano plazo, la probabilidad de un estancamiento económico generalizado en la región fue bajo. Ejemplos de esta dinámica son los siguientes:

1. Sonora: Benjamín Hill.

2. Nuevo León: el municipio de China, en el extremo oriente del estado.

3. Puebla: Ajalpan en la región de Tehuacán y Sierra Negra.

4. Oaxaca: Santiago Ixcuintepec (distrito Zacatepec en la región Sierra Norte).

5. Chiapas: Zinacantán (municipio contiguo a San Cristóbal en la región Altos Tzotzil-Tzeltal).

6. Tabasco: los municipios de Mascupana (donde se localiza Ciudad-PEMEX) y Jonuta.

7. Campeche: Hopelchén (zona arqueológica).

8. Yucatán: municipio de Chocholá, en el corredor Ciudad deCaCampeche-Mérida.

Tras esta primera exploración geográfica del crecimiento económico se observó que no hay una dinámica dominante (mapa 3). No obstante es posible inferir que existió un efecto de arrastre en el crecimiento de un municipio respecto a su región, de ahí que se registraron también conglomerados de municipios. Por ello, en segundo lugar se realizó un análisis detallado de éstos en el cual se mezclaron diferentes dinámicas territoriales. A continuación se presenta la definición y conformación municipal de dos conglomerados que fueron los más representativos en el territorio, los cuales definen dos procesos en las dinámicas territoriales de crecimiento: el virtuoso y el vicioso; además se delinean algunas directrices para una política económica de corte regional.

\section{a) Procesos virtuosos en las dinámicas de crecimiento (AA y BA):} en estas regiones prevaleció un proceso virtuoso de crecimiento en las dinámicas territoriales, se favoreció el contagio por su contigüidad geográfica. Sin embargo, hubo municipios que registraron un estancamiento pese a que hubo otros en crecimiento que lo rodearon. Por ello una política económica regional rendirá buenos frutos, en los municipios estancados (BA) y contiguos a otros de crecimiento virtuoso (AA), en la medida en que ésta favorezca la integración de la región a través de, por ejemplo, infraestructura en comunicaciones y transporte. Algunas regiones son: 
1. Chihuahua: la región entre Creel (Boycona) Chihuahua y Choix Sinaloa en la Sierra Madre se caracteriza por un proceso virtuoso, en donde el crecimiento de estos municipios está encapsulado, por lo que no hay contagio a los municipios vecinos como Guachochi, Batopilas y Guazapares. Al este se localiza Huejotitán y El Tule, este último con una dinámica tipo AA y cercano a Parral, también con un proceso virtuoso para la región.

2. San Luis Potosí: el proceso se observa en dos regiones. En el centro, el caso de la ZM de San Luis Potosí-Soledad de Graciano Sánchez, el municipio de la capital aún no ha logrado contagiar su crecimiento a los municipios que lo rodean. Mientras que en el Altiplano Potosino, al norte del estado, lo mismo ocurre con el municipio de Matehuala y sus vecinos.

3. Aguascalientes: al norte de la ciudad-capital se registró este proceso en la dinámica de crecimiento, en donde el crecimiento del municipio de Rincón de Romos no se dispersó a sus vecinos, como en el caso de Pabellón de Arteaga que continuó con un bajo crecimiento.

4. Hidalgo: con una dinámica virtuosa de crecimiento encontramos las regiones de Actopan, Tizayuca y Huejutla; mientras que con una dinámica virtuosa de estancamiento se localizan las regiones de Atotonilco, Pachuca y Tula; por ello esta región registró un proceso virtuoso.

5. Puebla: el Valle de Atlixco y Matamoros -con centro en Izúcar de Matamoros- tienen una dinámica virtuosa de crecimiento y estancamiento.

6. Oaxaca: varios municipios de la región mixteca de Oaxaca, localizados en los distritos de Tlaxiaco y Teposcolula, también registraron un proceso virtuoso, éste se registró también en los distritos de Ixtlán (región Sierra Norte) y Miahuatlán (región Sierra Sur), en la región Costa de Oaxaca, y en municipios de los distritos de Pochutla y Miahuatlán.

7. Veracruz: municipios de la ZM de Minatitlán y otros contiguos se encuentran inmersos en este tipo de procesos en su dinámica de crecimiento regional.

\section{b) Procesos viciosos en las dinámicas de crecimiento (BB y $\mathrm{AB}$ ):} registrados en regiones más o menos integradas donde, pese a que existen dinámicas de crecimiento en algunos municipios, no prevaleció la propagación del crecimiento sino el contagio del estancamiento (BB). Por ello, una política regional que estimule el 
crecimiento será infructífera ante la prevalencia de economías de enclave en la región $(\mathrm{AB})$. Es decir, si bien se requieren inversiones dirigidas para romper el círculo vicioso en municipios $\mathrm{BB}$, en los casos en que el crecimiento esté encapsulado $(\mathrm{AB})$ las inversiones deben acompañarse de una política regional de encadenamiento productivo, la cual integre a los municipios vecinos con el enclave en la búsqueda de un crecimiento regional. En esta situación se encuentran los siguientes municipios:

1. Sonora: en la región Costa, los municipios de Hermosillo, Guaymas y Cajeme registraron una dinámica BB que contrasta con la $\mathrm{AB}$ de municipios rurales como San Javier, Villa Pesqueira y Sahuaripa. Hacia la frontera, en la zona limítrofe con Chihuahua, se encuentra una extensa región con una dinámica de estancamiento (BB) integrada por Imuris, Santa Cruz, Cananea, Naco, Bacoachi, Fronteras, Nacozari de García, Bavispe, Huachineras y Agua Prieta, mientras que Nacozari de García funcionó como el enclave minero de la región $(\mathrm{AB})$.

2. Chihuahua: en la frontera limítrofe con Sonora la región de Janos $(\mathrm{BB})$ y Casas Grandes $(\mathrm{AB})$ registró un proceso vicioso; además en la región de municipios contiguos a Ciudad Cuauhtémoc se observó también este proceso donde el municipio $\mathrm{AB}$ o enclaves (Santa Isabel) es contiguo a los BB (Cuauhtémoc, Cusihuiriachi, Dr. Belisario Domínguez).

3. Jalisco: al sur de la ZM de Guadalajara, en la zona del Lago de Chapala y de la Laguna de Sayula (Jocotepec, Ixtlahuacán de los Membrillos, Zacoalco de Torres, Atoyac y Tuxcueca), se observa un proceso vicioso de crecimiento; solo los municipios de Jocotepec y Atoyac no lograron encauzar la dinámica de crecimiento de sus municipios vecinos.

4. Michoacán: con este proceso se encuentra también la ZM de Morelia y además de los municipios vecinos del occidente como Quiroga (entrada a la región del Lago de Pátzcuaro-Zirahuén), Chucándiro y Huaniqueo de Morales (en la carretera MoreliaJiquilpan-Guadalajara), éste último ha registrado un crecimiento alto en comparación a sus vecinos.

5. Puebla: este proceso se registró en la región de Huachinango, en la sierra norte del estado de Puebla; en los municipios al oriente del área metropolitana de Puebla (San Andrés Cholula y Santa Isabel Cholula) y en aquéllos donde se asienta el área urbana de San Martín Texmelucan que conforman el extremo oriente de la ZM de Puebla-Tlaxcala; así como en la región 
mixteca-sur del estado más los municipios contiguos a esta zona en la montańa de Guerrero y otros en el distrito de Huajapan, Oaxaca.

6. Oaxaca: algunos municipios en el distrito de Ocotlán en la región Valles Centrales de Oaxaca (al sur de la ciudad de Oaxaca).

7. Veracruz: municipios al norte de la región Córdoba-Orizaba registraron también un proceso vicioso en su dinámica de crecimiento.

8. Tamaulipas: en los límites entre Veracruz y Tamaulipas se conformó una región con un proceso vicioso. Ésta se integra por la ZM de Altamira-Tampico-Madero (Tamaulipas) y Pueblo Viejo (Veracruz), aquí la actividad económica de Tampico se ha contagiado hacia Pueblo Viejo, pero no hacia Altamira.

9. Tabasco: Paraíso registró un crecimiento alto en relación con sus vecinos (enclave petrolero), el cual no ha podido disgregarse a su alrededor; por ello municipios como el de Jalpa de Méndez continuó registrando un bajo crecimiento.

Este análisis revela que las dinámicas regionales continúan siendo significativamente importantes, además permitió identificar los procesos que prevalecen en tales dinámicas, ya sea virtuosa $(A A+B A)$ o viciosa $(B B+A B)$. No obstante, se observa un efecto neto perverso ya que estos municipios no están concentrados en una zona en particular del país, sino que están distribuidos aleatoriamente en el territorio: municipios con alguna dinámica de estancamiento (BB o BA) están espacialmente correlacionados (i.e. contiguos) a clústeres o enclaves que registraron una dinámica de crecimiento (AA o AB). Lo anterior sugiere que en la década 1999-2009 no sólo el crecimiento se ha propagado hacia áreas vecinas, sino también el estancamiento económico.

\subsection{La dimensión espacio-temporal}

Para el análisis de las dinámicas territoriales en el tiempo se propuso relacionar el crecimiento económico en un año $t$ en un municipio en función a los valores observados entre los municipios vecinos en el año t-1 (análisis bivariado). Así, comparando el crecimiento económico en el quinquenio 2004-2009 y el observado en los municipios vecinos durante el quinquenio de 1999-2004, el estadístico global de la I de Moran arrojó el valor de -0.0432 (con un p-value de 0.0001); es decir, un patrón aleatorio de autocorrelación espacio-temporal negativo. Este patrón muestra una tendencia a la agrupación de municipios con valores disími- 
les en diferentes regiones del territorio nacional, este resultado sugiere que un municipio con alto crecimiento económico en el segundo quinquenio estuvo rodeado de municipios con un crecimiento bajo en el primero.

Para indagar sobre la heterogeneidad espacio-temporal de las dinámicas de crecimiento, el mapa LISA bivariado (mapa 3) muestra los agrupamientos del análisis local de autocorrelación espacial (i.e. AA, BB, BA y $A B)$. Sin embargo, para señalar cuál fue el comportamiento de las dinámicas previamente identificadas se clasificó a los municipios en los grupos $\mathrm{AA}$ y $\mathrm{BB}$ como aquellos que experimentaron una persistencia al crecimiento o una persistencia al estancamiento, respectivamente, pues en ambos quinquenios se observó el mismo patrón y por tanto se generó una tendencia en la década.

Ejemplos de municipios AA son Comala, al norte de la ciudad de Colima; Rincón de Romos, en Aguascalientes; municipios de la Husteca Hidalguense; Acambay en el Estado de México; Nopalucan en Tlaxcala; Acatepec en Guerrero; la ciudad de Oaxaca; Boca del Río, la ZM de Coatzacoalcos y Chinameca y Zaragoza (en la ZM de Minatitlán) en Veracruz. También con esta tendencia al crecimiento se encuentra la región petrolera de Jonuco en Tabasco y Carmen en Campeche; así como Ocotepec en Chiapas e Izamal en Yucatán. Mientras que ejemplos de municipios BB son La Cruz, al sureste de Chihuahua; Acuña y Guerrero en Coahuila; Villa González Ortega y Villa Hidalgo en Zacatecas; Ixtlahuacán de los Membrillos, Tonalá y El Salto en el extremo sur oriental de la ZM de Guadalajara; Tehuacán en Puebla; y San Pedro Totolapa, Santa María Quiegolani y San Pedro Ocotepec en Oaxaca.

Sin embargo, a través del tiempo puede encontrarse también una "resiliencia territorial" en las dinámicas de crecimiento, ${ }^{6}$ definida ésta como la tendencia de algunos municipios a mantener en el tiempo cierta dinámica de crecimiento, incluso cuando han permanecido rodeados de municipios con un comportamiento diferente. Así, la resiliencia al estancamiento $(\mathrm{AB})$ se registró cuando las economías municipales con altos niveles de crecimiento en 2004-2009 se encontraron rodeadas geográficamente de municipios con niveles bajos de crecimiento en 19992004. Estos municipios tuvieron la "habilidad" de hacer frente a su entorno y no "contagiarse" de situaciones que provocasen un lento crecimiento económico o una crisis. En cambio, los municipios con bajo crecimiento en el periodo 2004-2009 estuvieron rodeados de municipios

\footnotetext{
${ }^{6}$ La resiliencia es entendida como la capacidad de sobreponerse a resultados adversos (Briguglio et al., 2009). Es decir, cuando una economía ha sido expuesta a choques exógenos (e.g. de la apertura económica, de las condiciones estructurales de la economía u otros que pueden constituirse en una desventaja para el desarrollo económico) su capacidad de hacerles frente se le conoce como resiliencia o capacidad de afrontamiento.
} 
con alto crecimiento (BA) en el periodo 1999-2004, por lo que registran una resiliencia al crecimiento, esto es, las economías municipales se resistieron a crecer a un ritmo similar o superior al de sus vecinos.

Al comparar los valores del crecimiento económico -rezagado temporal y espacialmente- se observó que el fenómeno de resiliencia al crecimiento (AB) está presente en un mayor número de municipios (mapa 3). $\mathrm{Si}$ bien se pueden registrar municipios en cada uno de estos agrupamientos, el número de municipios aislados es escaso, dando lugar a regiones conformadas por municipios $\mathrm{AA}$ y $\mathrm{BA}$, o bien, $\mathrm{BB}$ y $\mathrm{BA}$, en los cuales existió temporalmente una resiliencia al crecimiento o una resiliencia al estancamiento, respectivamente. Así, observando el crecimiento por quinquenio y la localización de los municipios, las tendencias regionales registradas fueron las siguientes:

a) Resiliencia al crecimiento (AA y BA): con el tiempo, los municipios en estas regiones registraron un crecimiento estable y positivo, lo que aseguró a sus habitantes mejores niveles de desarrollo económico en el largo plazo, pues el crecimiento se mantuvo en el primero y segundo quinquenio de la década de estudio. Sin embargo, hubo municipios con alto crecimiento en ambos quinquenios (AA), los cuales conformaron un clúster de crecimiento, estos se localizaron contiguos a municipios que no lograron mejorar sus condiciones de crecimiento en el periodo 2004-2009 pese a que estaban rodeados de municipios que registraron una dinámica de crecimiento favorable en el periodo 1999-2004 (BA). En esta situación se encontraron regiones que se localizan en el centro y sur del país, como las que a continuación se señalan:

1. Jalisco: en municipios de la Sierra de Amula, como El Grullo y Ejutla, se registró una tendencia al crecimiento, mientras que Tenamaxtlán mostró resiliencia.

2. Hidalgo: la ZM de Pachuca de Soto, además de los municipios de Mineral del Chico y Actopan, fue una región que mostró una tendencia al crecimiento (Mineral del Chico, San Agustín Tlaxiaca, Zapotlán y Zempoala), pero también hubo municipios resilientes al crecimiento (Mineral del Monte, Mineral de la Reforma y Actopan).

3. Tlaxcala: en el extremo norte de la de la ZM de Tlaxcala-Apizaco, los municipios de Tetla de la Solidaridad, Terrenate y Muñoz de Domingo Arenas registraron una tendencia al crecimiento, mientras que Tlaxco fue resiliente al crecimiento. 
4. Puebla: en la mixteca poblana, incluyendo a Izúcar de Matamoros al sur de la región del Valle de Atlixco y Matamoros, prevalece una tendencia al crecimiento entre los municipios; con excepción de Tehuitzingo, Piaxtla y San Pedro Yeloixtlahuaca que muestran una resiliencia al crecimiento. Hacia el oriente del estado en la región Serdán, el municipio de Chalchicomula de Sesma (Ciudad Serdán) mostró una resiliencia al crecimiento en el segundo quinquenio pese al alto crecimiento de su vecino, el municipio de Esperanza.

5. Oaxaca: buena parte de la mixteca oaxaqueña puede clasificarse con este tipo de tendencia, particularmente en el distrito de Huajuapan donde son numerosos los municipios que registraron una tendencia hacia la resiliencia y pocos los que conservaron una tendencia al crecimiento en ambos quinquenios. Son varios los municipios que forman un conglomerado de municipios resilientes en donde confluyen los distritos de Juxtlahuaca, Tlaxiaco, Teposcolula y Nochixtlán. También, en el distrito de Coixtlahuacan la mayoría de los municipios presentaron una tendencia a la resiliencia. En la región Cañada, conformada por los distritos de Cuicatlán y Teotitlán, se formó otro agrupamiento de municipios resilientes; éste quedó conformado por los municipios de San Pedro Teutila, Santa María Tlalixtac y San Francisco Chapulapa (distrito de Cuicatlán), así como San Bartolomé Ayautla y San Juan Coatzospam (distrito de Teotitlán). En la región Costa, los municipios de San Agustín Loxicha (distrito de Pochutla), Santa Lucía Miahuatlán y San Marcial Ozolotepec mostraron una tendencia AA, mientras que San Mateo Río Hondo (distrito de Miahuatlán) mostró resiliencia.

Se registraron casos aislados donde un municipio o grupo de municipios presentaron resiliencia al crecimiento (BA), tal es el caso de Chihuahua, donde varios municipios al norte (Ascensión, Nuevo Casas Grandes, Galeana, Ignacio Zaragoza, Buenaventura y Ahumada) no lograron contagiarse de la prosperidad de sus vecinos con el paso del tiempo. También se encontraron otros casos con esta tendencia en Nuevo León (municipios de Allende y General Terán), en la ZM de Zacatecas y en el municipio de Tabasco en el estado de Zacatecas, así como en el municipio de Asientos (Aguascalientes).

Hacia el centro del país, los municipios de Luvianos, Temascaltepec y Morelos en el Estado de México; Cuautla y Ocuituco en Morelos; Coyomeapan en la región de Tehuacán y Sierra Negra de Puebla fueron 
resilientes al crecimiento; al suroeste, los municipios de Coataxtla y Manlio Fabio Altamirano al oeste de la ZM de Veracruz-Boca del Río, así como municipios de la región Valles Centrales en Oaxaca, en el distrito de Tlacolula (Teotitlán del Valle, San Pablo Villa de Mitla y Tlacolula de Matamoros) y San Andrés Ixtlahuaca en el distrito Centro.

b) Resiliencia al estancamiento (BB y AB): en estas regiones la persistencia de los municipios $\mathrm{BB}$ es negativa, ya que en el tiempo la tendencia al estancamiento se mantuvo, por esta razón, en diez años no se logró generar una tendencia estable de crecimiento. Sin embargo, a nivel regional, pese a ser una zona integrada por municipios de bajo crecimiento o económicamente deprimidos, coexisten en el territorio enclaves económicos en algunos municipios de la región (AB). Pese al crecimiento bajo y persistente de sus vecinos en la primera mitad de la década, éstos lograron crecer a una tasa superior en el segundo quinquenio; de ahí que hayan sido resilientes a una desaceleración de su crecimiento o estancamiento. Ejemplos de estas regiones, localizadas comúnmente en el norte del país, son:

1. Sonora: varios municipios al noreste del estado como Cananea, Naco, Bachoachi, Fronteras, Agua Prieta conforman una región estancada donde el municipio de Nacozari de García registró tasas de crecimiento altas en el periodo 2004-2009. Hacia el centro-sur del estado los municipios de Cajeme, Rosario, Yécora, Suaqui Grande y Bacanora constituyen la región estancada mientras Onavas, San Javier y Villa Pesqueira son municipios $\mathrm{AB}$.

2. Chihuahua: en la región del centro sur de la Sierra Madre en Chihuahua los municipios de Urique, Uruachi, Chinipas, Moris y Temosachic son resilientes al estancamiento, mientras que solo Maguarichi es BB.

3. Hidalgo: en la Sierra Alta se registró este patrón en los municipios de Xochicoatlán, Eloxochitlán y Molango de Escamilla, este último con resiliencia al estancamiento.

4. Estado de México: al oriente de la ZM de la Ciudad de México, los municipios de Ixtapaluca y Chimalhuacán registraron una tendencia hacia el estancamiento, no obstante Texcoco y La Paz mostraron una resiliencia al estancamiento en el segundo quinquenio.

5. Puebla: en Sierra Norte del estado los municipios de Xicotepec, Jopala, Amixtlán y Ahuacatlán registraron una resiliencia al 
estancamiento; mientras que Huachinango, Tlapacoya y Coahuitlán mostraron una tendencia al estancamiento (BB).

6. Oaxaca: en la Región Sierra Sur varios municipios del distrito Sola de Vega registraron una resiliencia al estancamiento (AB), e.g. Villa Sola de Vega, Santiago Minas, Santo Domingo Tejomulco, Santa María Zaniza, además de San Antonio Huitepec (distrito de Zaachila, región Valles Centrales) y San Pedro Juchatengo (distrito de Juquila, región Costa); mientras que en Santa Cruz Zenzontepec y Santiago Textitlán hubo persistencia al estancamiento. Por el contrario, en municipios de la región Valles Centrales dominaron municipios BB como Ejutla de Crespo (distrito de Ejutla), San José del Progreso, San Pedro Taviche y Santo Tomás Jalieza (distrito de Ocotlán), además de San Juan Teitipac (distrito de Tlacolula); éstos coexistieron con otros AB,por ejemplo San Martín de los Cansecos (Ejecutla), San Jerónimo Taviche y San Baltazar Chihicapam (Ocotlán).

7. Chiapas: en la región de la Selva Lacandona, el municipio de Ocosingo registró una tendencia al estancamiento, mientras que una extensa región mostró resiliencia; esta última se conformó por los municipios de Chilón, Yajalón, Sitalá, Tumbalá (región de Tulijá Tzeltal Chol), San Juan Cancuc y Pantelhó (región Altos Tzotzil-Tzeltal), Simojovel, El Bosque, Ixtapa, Pueblo Nuevo Solistahuacán (región de Bosques) y Santiago del Pinar y Larráinzar (región de San Cristóbal de las Casas). Otros municipios BB fueron Chalchihuitán en los Altos y Pueblo Nuevo Solistahuacán en región Bosques.

8. Yucatán: al oriente de la ZM de Mérida, en municipios contiguos a Motul (Tixkokob, Muxupip, Motul, Bokobá en la región henequenera) presentan una resiliencia al estancamiento, mientras que Tahmek fue BB. Además, en el extremo sur del estado se conforma una región en donde Peto y Cantamayec presentan una tendencia BB, mientras que Tzucacab, Tixmehuac y Tahdziu fueron municipios resilientes al estancamiento.

Se registran casos aislados de municipios que pese a no crecer en el primer quinquenio lo hicieron en el segundo, por lo que mostraron una resiliencia al estancamiento (AB). Ejemplo de ello son municipios al norte de Durango (Ocampo), al norte del ZM de Monterrey (Minas y Salinas Victoria); el municipio de Xilitla al sur de la huasteca potosina; Pacula y Jacala de Ledezma en la región Sierra Gorda de Hidalgo; Tultitlán y San Mateo Atenco en el Estado de México; Nativitas Tlaxcala en la ZM de 
Puebla-Tlaxcala; en municipios al norte de la ZM de Xalapa (Coacoatzintla) y al sur de Coatepec (Teocelo, Cosautlán de Carvajal y Ayahualulco) en Veracruz; Mapastepec en el Istmo Chiapaneco. En Oaxaca estos casos ocurren en municipios de la Región Sierra Sur: en el distrito de Miahuatlán (San Francisco Ozolotepec, San Juan Ozolotepec y San Pedro Mixtepec) y en el de Yautepec (Nejapa de Madero, San Bartolo Yautepec y San Juan Juquila Mixes).

\section{Conclusiones}

El crecimiento económico es un fenómeno espacial por naturaleza y en este trabajo se presentó evidencia de que los municipios mantienen procesos de propagación del desarrollo económico que pueden explicarse, entre otros factores, por el propio arreglo espacial de las áreas geográficas. De ahí la necesidad de conocer el patrón espacial que explica dicho comportamiento e identificar las dinámicas territoriales del crecimiento y evaluar la dependencia y heterogeneidad espacial entre los municipios de México. Para ello, la metodología implicó un enfoque espacial en la identificación de patrones y agrupamientos de municipios que, a través de un análisis de autocorrelación local, permitió categorizar similitudes y diferencias en el crecimiento económico entre municipios vecinos.

En primer lugar, el estudio nos llevó a conocer más sobre los patrones de crecimiento económico en el país, particularmente, el análisis exploratorio confirmó estadísticamente la presencia de la dependencia espacial; este análisis sugirió un patrón de distribución aleatorio que permite inferir la existencia de un efecto de arrastre en el crecimiento de un municipio, ya sea positivo o negativo, a consecuencia de la interacción con sus vecinos. Además se mostró la heterogeneidad de este fenómeno en el territorio, es decir, el efecto que tiene la contigüidad geográfica en el crecimiento es diferenciado, lo que es más evidente en municipios de entidades del noroeste y centro-sureste del país.

En segundo lugar, las técnicas utilizadas permitieron identificar la localización y el alcance de los clústeres geográficos y enclaves productivos en el territorio nacional a través de un cambio en el abordaje metodológico que analizó la autocorrelación espacial a escala local. En función de ello y de la heteregeneidad del fenómeno en el territorio se establecieron cuatro dinámicas de crecimiento en el periodo 1999-2009. De la misma manera, en una perspectiva regional se distinguieron dos procesos: uno virtuoso, característico de regiones en el centro y sur del país, y uno vicioso, que se presentó en el norte de México y en menor medida en municipios de estados costeros del pacífico y golfo. Con ello se mostró que las dinámicas regionales continúan siendo significativamente impor- 
tantes, pues no se puede establecer el dominio de una sola dinámica territorial.

Además, en tercer lugar, como un elemento innovador en el estudio, se analizó simultáneamente la dimensión temporal y geográfica para conocer la heterogeneidad en la distribución del crecimiento; con ello se pretendió un enfoque espacial dinámico, más que uno espacial estático del fenómeno estudiado. Se identificaron también cuatro tendencias de las dinámicas en el tiempo, a saber, persistencia y resiliencia de los municipios hacia el crecimiento o el estancamiento. Con lo anterior se mostró evidencia de que el crecimiento en el segundo quinquenio para un municipio estuvo influenciado por el crecimiento de los vecinos en el primer quinquenio, pero siguiendo un patrón aleatorio en el territorio. Sin embargo, tras una década de transformaciones económicas la evidencia sugiere que cada vez son menos los municipios con similitudes en sus dinámicas de crecimiento, lo cual apunta hacia un campo aún desconocido de dinámicas regionales no-lineales, y por tanto irregulares, de crecimiento económico.

No debe olvidarse que, en cuarto lugar, el crecimiento se contagia hacia áreas más extensas del territorio en función de las interdependencias existentes, incluida la que se despliega en el propio espacio geográfico; por ello debemos concluir que también una dinámica de estancamiento, así como de resiliencia al crecimiento o al estancamiento son procesos que se contagian entre municipios vecinos. De ahí que la categorización presentada es útil para analizar el crecimiento económico desde una nueva perspectiva, donde el espacio se conceptualiza como un recurso económico que genera ventajas estáticas y dinámicas, dejando de lado un enfoque tradicional en donde el espacio no importa.

Lo anterior permite argumentar la pertinencia de una política económica de corte regional. Este punto de vista es importante pues presenta evidencia sobresaliente para efectos de planeación regional, especialmente en un contexto reciente donde las politicas implementadas han sido espacialmente "ciegas". Así, el patrón de propagación del crecimiento y los efectos de la autocorrelación espacial en el territorio permiten reforzar la necesidad de politicas regionales, pese a la tendencia de una población cada vez más urbana.

En quinto lugar, cabe recalcar que el análisis exploratorio de datos espaciales ayudó a conocer las dinámicas de propagación de crecimiento económico presente entre los municipios de México, es decir, la autocorrelacion espacial global y local. Por ello conviene plantearse la verificación a futuro de la hipótesis de convergencia, es decir, comprobar si los municipios pobres crecen más rápido que los municipios ricos. Si para ello utilizamos un enfoque espacial, el cual integre los efectos y procesos es- 
paciales en sus estimaciones, se puede verificar la presencia de regímenes espaciales en el crecimiento económico y evaluar si las economías geográficamente cercanas a un municipio afectan este proceso, inclusive ante la presencia de condiciones iniciales diferenciadas.

Finalmente, conocer qué mecanismos o políticas regionales se llevaron a cabo en los municipios y regiones identificadas con alguna dinámica territorial de crecimiento resulta una tarea interesante que quedará pendiente, pese a que son sólo algunos municipios los que están jugando un rol destacado en el crecimiento regional de México. Se requiere pues de un análisis más exhaustivo para conocer la naturaleza espacial del crecimiento económico y entender las dinámicas en una región en particular. No obstante, esperamos que la información aquí vertida sea de utilidad para académicos, estudiantes e involucrados en la toma de decisiones y genere al mismo tiempo interés por conocer los procesos económicos territoriales sobre los cuales deben ejecutarse políticas regionales.

\section{Fuentes consultadas}

Abreu, Maria, Henri De Groot y Raymond Florax (2005), "Space and growth: a survey of empirical evidence and methods", Région et Développement, vol. 21, Tinbergen Institute, Ámsterdam, Países Bajos, pp. 13-44.

Aguayo, Francisco y Carlos Salas (2002), "Reestructuración y dinámica del empleo en México, 1980-1998”, Región y Sociedad, XIV (25), El Colegio de Sonora, Hermosillo, México, pp. 3-62.

Anselin, Luc (2007), "Spatial econometrics in RSUE: retrospect and prospect”, Regional Science and Urban Economics, 37 (4), Elsevier, Amsterdam, Holanda, pp. 450-456.

Anselin, Luc (2003), "Spatial econometrics", en Badi Baltagi (ed.), $A$ companion to theoretical econometrics, Blackwell Publishing, New Jersey, Estados Unidos de América, pp. 310-330.

Anselin, Luc (2000), "Computing environments for spatial data analysis", Journal of Geographical Systems, 2 (3), Springer, Heidelberg, Alemania, pp. 201-220.

Anselin, Luc (1988), Spatial Econometrics: methods and models, Springer Netherlands, Ámsterdam, Países Bajos. 
Anselin, Luc, Sanjeev Sridharan y Susan Gholston (2007), "Using exploratory spatial Data Analysis to leverage social indicator databases: the discovering of interesting patterns", Social Indicators Research, 82 (2), Springer, Michigan, Estados Unidos de América, pp. 287-239.

Barnes, Trevor (2002), "Inventing Anglo-American Economic Geography 1889-1960", in Eric Sheppard y Trevor Barnes (eds.), A companion to Economic Geography, Blackwell Publishing, Oxford, Inglaterra, pp. 11-26.

Barnes, Trevor (2001), "Retheorizing Economic Geography: from the quantitative revolution to the cultural turn", Annals of the Association of American Geographers, 91 (3), American Association of Geographers, Taylor \& Francis Group, Washington, Estados Unidos de América, pp. 546-565.

Barro, Robert y Xavier Sala-i-Martin, (2004), Economic Growth, The MIT Press, Cambridge, Massachusetts, Estados Unidos de América.

Baumont, Catherine, Cem Ertur y Julie Le Gallo (2004), "Spatial analysis of employment and population density: the case of the agglomeration of Dijon 1999", Geographical Analysis, 36 (2), The Ohio State University, Wiley-Blackwell, Ohio, Estados Unidos de América, pp. 146-176.

Boyce, David (2004), "A short history of the field of regional science", in Raymond Florax and Davis Plane (eds.), Fifty years of Regional Science, Regional Science Association International, SpringerVerlag, Berlín, Alemania, pp. 31-57.

Briguglio, Lino, Cordina Gordon, Nadia Farrugia, y Stephanie Vella (2009), "Economic vulnerability and resilience: concepts and measurements", Oxford Development Studies, 37 (3), Oxford Department of International Development, Routledge, Oxford, Inglaterra, pp. 229-247.

Cliff, Andrew y Keith Ord (1970), "Spatial autocorrelation: a review of existing and new measures with applications", Economic Geography, vol. 46, Clark University, Worcester, Estados Unidos de América, pp. 269-292. 
Durlauf, Steven, Paul Johnson y Jonathan Temple (2005), "Growth econometrics”, en Philippe Aghion y Steven Durlauf (eds.), Handbook of Economic Growth, vol. 1, Elsevier, Ámsterdam, Holanda, pp. 555-677.

Ertur, Cem y Julie Le Gallo (2009), "Regional growth and convergence: Heterogeneous reaction versus interaction in spatial econometric approaches", en Roberta Capello y Peter Nijkamp (eds.), Handbook of regional growth and development theories, Edward Elgar Publishing, Massachusetts, Estados Unidos de América, pp. 374-388.

Fingleton, Bernard y Enrique López-Bazo, (2006), "Empirical growth models with spatial effects", Papers in Regional Science, 85 (2), Regional Science Association International, Blackwell Publishing, Oxford, Inglaterra, pp. 177-198.

Florax, Raymond y Peter Nijkamp, (2003), Misspecification in linear spatial regression models, discussion paper TI 2003-081/3, Tinbergen Institute of Economic Research, Amsterdam, Holanda.

Florax, Raymond y David Plane (2004), "Introducing the brightest of dawns: Regional Science in papers", en Florax Raymond y David Plane (eds.), Fifty years of Regional Science, Regional Science Association International-Springer Verlag, Berlín, Alemania, pp. 5-28.

Fujita, Masahisa y Mori Tomoya (2005), "Frontiers of the New Economic Geography", Papers in Regional Science, 84 (3), Regional Science Association International, Blackwell Publishing, Oxford, Inglaterra, pp. 377-405.

Geary, Robert (1954), "The contiguity ratio and statistical mapping”, The Incorporated Statistician, 5 (3), Royal Statistical Society, Londres, Inglaterra, pp. 115-145.

Getis, Arthur y Keith Ord, (1992), "The analysis of spatial association by use of distance statistics", Geographical Analysis, 24 (3), The Ohio State University, Ohio, Estados Unidos de América, pp. 189-206.

Griffith, Daniel (2003), Spatial autocorrelation and spatial filtering, Springer-Verlag, Berlin, Alemania. 
Goodchild, Michael, Luc Anselin, Richard Appelbaum y Barbara Herr Harthorn (2000), "Toward spatially integrated social science", International Regional Science Review, 23 (2), Sage publications, Thousand Oaks, Estados Unidos de América, pp. 139-159.

Hoover, Edgar M. y Frank Giarratani (1984), An Introduction to Regional Economics, Alfred A. Knopf, Nueva York, Estados Unidos de América.

Inegi (Instituto Nacional de Estadística y Geografía) (2015a), Censos Económicos 1999, Inegi, Aguascalientes, México, <http://www3. inegi.org.mx/sistemas/saic/?evento=1999>, 12 de marzo de 2015.

Inegi (Instituto Nacional de Estadística y Geografía) (2015b), Censos Económicos 2004, Inegi, Aguascalientes, México, <http://www3. inegi.org.mx/sistemas/saic/?evento=2004>, 12 de marzo de 2015.

Inegi (Instituto Nacional de Estadística y Geografía) (2015c), Censos Económicos 2009, Inegi, Aguascalientes, México, <http://www3. inegi.org.mx/sistemas/saic/>, 12 de marzo de 2015.

Inegi (Instituto Nacional de Estadística y Geografía) (2015d), Censo de Población y Vivienda 2010, Inegi, Aguascalientes, México, <http:// www.inegi.org.mx/sistemas/olap/Proyectos/bd/censos/cpv2010/ PT.asp?s=est\&c=27770\&proy=cpv10_pt>, 12 de marzo de 2015.

Inegi (Instituto Nacional de Estadística y Geografía) (2015e), II Conteo de Población y Vivienda 2005, Inegi, Aguascalientes, México, $<$ http://www.beta.inegi.org.mx/proyectos/ccpv/2005/>, 12 de marzo de 2015.

Inegi (Instituto Nacional de Estadística y Geografía) (2015f), XII Censo General de Población y Vivienda 2000, Inegi, Aguascalientes, México, <http://www.beta.inegi.org.mx/proyectos/ccpv/2000/ default.html >, 12 de marzo de 2015 .

Isard, Walter (2003), History of Regional Science and the Regional Science Association International. The beginnings and early history, SpringerVerlag, Berlín, Alemania.

Krugman, Paul (1998), Development, geography, an economic theory, MIT Press, Cambridge, Londres, Inglaterra. 
Moran, Patrick (1948), "The interpretation of statistical maps", Journal of the Royal Statistical Society, Series B. Methodological, 10 (2), Royal Statistical Society, Londres, Inglaterra, pp. 243-251.

Ponsard, Claude (1983), History of Spatial Economic Theory, SpringerVerlang Berlín Heidelberg, Nueva York.

Rey, Serge y Sastré-Gutiérrez, Myrna (2010), “Interregional inequality dynamics in Mexico", Spatial Economic Analysis, 5 (3), Regional Studies Association, Routledge, Abingdon, Reino Unido, pp. 277-298.

Rey, Serge (2004), "Spatial analysis of regional income inequality", en Michael F. Goodchild y Donald G. Janelle (eds.), Spatially integrated social science, Oxford University Press, Oxford, Inglaterra, pp. 280-299.

Rey, Serge y Luc Anselin (2000), "Regional science publication patterns in the 1990", International Regional Science Review, 23 (4), Sage publications, Thousand Oaks, Estados Unidos de América, pp. 323-344.

Sastré-Gutiérrez, Myrna y Serge Rey (2008), "Polarización espacial y dinámicas de la desigualdad intrerregional en México", Problemas del Desarrollo, 39 (155), Universidad Autónoma de México, Ciudad de México, México, pp. 182-204.

Tobler, Waldo (1970), "A computer movie simulating urban growth in the Detroit region”, Economic Geography, vol. 46, Clark University, Worcester, Estados Unidos de América, pp. 234-240.

Recibido: 18 de junio de 2016. Corregido: 23 de diciembre de 2016. Aceptado: 25 de abril de 2017.

Liz Ileana Rodríguez-Gámez. Doctora en Geografía por la Universidad de Arizona, Estados Unidos. Actualmente se desempeña como profesorainvestigadora de El Colegio de Sonora, profesora de la Facultad de Economía de la Universidad de Sonora. Es candidato a investigador nacional 
del Sistema Nacional de Investigadores. Sus actuales líneas de investigación se centran en tópicos de geografía económica y econometría espacial. Entre sus últimas publicaciones destacan: "Demanda y provisión de trasporte público en Ciudad Obregón, Sonora”, Región y Sociedad, XXVIII (67), El Colegio de Sonora, Hermosillo, México, pp. 243-275 (2016); "Employment density in Hermosillo, Mexico (1999-2009): A Spatial Econometric Approach of Local Parameters", Selected papers of Beijing Forum 2012, Peking University Press, Beijin, China, pp. 203-219 (2013); en coautoría, "Spatial distribution of employment in Hermosillo, 19992004”, Urban Studies, 49 (16), Sage Publications, Thousand Oaks, Estados Unidos de América, pp. 3663-3678 (2012).

José Antonio Cabrera-Pereyra. Doctorando en Geografía en la Universidad de Arizona, Estados Unidos. Entre sus publicaciones destacan, en coautoría: “Trabajo calificado, especialización y productividad laboral urbana en la frontera norte de México: un análisis de panel de efectos mixtos", Investigación Económica, LXXIII (287), Universidad Nacional Autónoma de México, Ciudad de México, México, pp. 89-119 (2014). 